\title{
Energy Moment Method Applied to Nuclear Quadrupole Splitting of Nuclear Magnetic Resonance Lines
}

\section{Frank, V}

Published in:

Journal of Chemical Physics

Link to article, DOI:

$10.1063 / 1.1732939$

Publication date:

1962

Document Version

Publisher's PDF, also known as Version of record

Link back to DTU Orbit

Citation (APA):

Frank, V. (1962). Energy Moment Method Applied to Nuclear Quadrupole Splitting of Nuclear Magnetic Resonance Lines. Journal of Chemical Physics, 37(1), 148-149. https://doi.org/10.1063/1.1732939

\section{General rights}

Copyright and moral rights for the publications made accessible in the public portal are retained by the authors and/or other copyright owners and it is a condition of accessing publications that users recognise and abide by the legal requirements associated with these rights.

- Users may download and print one copy of any publication from the public portal for the purpose of private study or research.

- You may not further distribute the material or use it for any profit-making activity or commercial gain

- You may freely distribute the URL identifying the publication in the public portal

If you believe that this document breaches copyright please contact us providing details, and we will remove access to the work immediately and investigate your claim 
Considering a single spur, let $n_{e}$ be the number of radicals which escape recombination as a result of hydrogen produced in that spur. Then an increment of dose, $d R$, to the system will cause radicals within the spur to disappear as a result of hydrogen produced in other spurs; so

$$
-d R / d \log n=R_{0} n_{e} / n,
$$

where $R_{0}$ is the initial value of $d R / d \log n$. Assuming that a particular spur is affected only by the hydrogen produced after its formation the integration of (14) between the pertinent limits gives

$$
n_{0}=n_{e} R_{0} /\left(R_{0}+R_{T}-R\right)
$$

$R_{T}-R$ being the dose received after the formation of the spur. We now want $\bar{n}_{0}$, an average value for all the spurs produced throughout the whole radiation inter- val. Since $d R / R_{T}$ is the fraction of the total number of spurs produced in the increment $d R$ our desired average is

$$
\begin{aligned}
n_{0} / n_{e} & =\left(R_{0} / R_{T}\right) \int_{0}^{R T}\left[d R /\left(R_{0}+R_{T}-R\right)\right] \\
& =\left(R_{0} / R_{T}\right) \log \left[1+\left(R_{T} / R_{0}\right)\right] .
\end{aligned}
$$

An estimate of $\bar{n}_{0} / n_{e}$ which is independent of Eq. (16) is not possible at this time, though it seems that it should certainly be greater than the ratio of $N_{0}$ to the number of free radicals formed when radiation is done in the presence of iodine. This ratio (for $16 \mathrm{~J} / \mathrm{g}$ radiation) is $5.65 \times 10^{17} / 2.0 \times 10^{18}=0.28$. The most plausible results from Eq. (16) are obtained by assuming $\bar{n}_{0} / n_{e}=0.70$ for the $16 \mathrm{~J} / \mathrm{g}$ treatment. This requires values of 0.39 and 0.145 for the 64 and $256 \mathrm{~J} / \mathrm{g}$ treatments.

\title{
Energy Moment Method Applied to Nuclear Quadrupole Splitting of Nuclear Magnetic Resonance Lines
}

\author{
V. Frank \\ Physics Department, Technical University of Denmark, Copenhagen
}

(Received March 1, 1962)

\begin{abstract}
Expressions giving the sum of the energy values, raised to the second and third power, for a nucleus interacting with a static magnetic field and a static electric field gradient are derived. Several applications of this method for obtaining the values of the components of the electric field gradient tensor from observed NMR spectra are suggested.
\end{abstract}

\section{INTRODUCTION}

$\mathbf{T}$ IHE method usually applied in extracting the values of the components of the electric field gradient tensor $V_{i j}$ at the site of a nucleus in a single crystal from experimental magnetic resonance data proceeds as follows: The values of the transition frequencies $\nu$, determined as functions of the angles specifying the direction of the magnetic field with respect to some set of reference axes fixed in the crystal, are compared with certain theoretical expressions for the $\nu$ 's containing $V_{i j}$ as parameters. These expressions are approximate only, being derived by perturbation calculations. The state of the art has recently been reviewed by Cohen and Reif. ${ }^{1}$

There is, however, another method, known as the method of energy moments, which has the advantage of being exact. This method has been discussed by Brown and Parker ${ }^{2,3}$ in connection with the problem considered here. Their final results are only stated in

1 M. H. Cohen and F. Reif, Solid State Phys. 5, 321-438, (1957).

2 L. C. Brown and P. M. Parker, Phys. Rev. 100, 1764 (1955).

${ }^{3}$ P. M. Parker and L. C. Brown, Am. J. Phys. 27, 509 (1959). the system of principal axes of $V_{i j}$, and this system is very often unknown in advance. It is the purpose of this note to give a short derivation of formulas pertaining to the general situation.

\section{THEORY}

The Hamiltonian for the system is (in frequency units $)^{1}$

$$
\mathfrak{H C}=-\nu_{0} I_{p} n_{p}+\frac{1}{9} \alpha V_{i j} T_{i j}
$$

where

$$
\alpha=3 e Q / 2 I(2 I-1) h,
$$

and

$$
T_{i j}=\frac{3}{2}\left(I_{i} I_{j}+I_{j} I_{i}\right)-\delta_{i j} I_{s} I_{s}
$$

(summation over dummy indices implied).

Here $\nu_{0}$ is the unperturbed nuclear Larmor frequency in the applied magnetic field $H_{0}$. The direction of $H_{0}$ is specified by the components $n_{p}$ of a unit vector in an arbitrary system of rectangular axes of reference fixed in the crystal. $I_{p}$ and $V_{i j}$ are the components of the spin vector operator and of the electric field gradient tensor, respectively, in this coordinate system. Finally, 
$Q$ is the quadrupole moment of the nucleus, and $I$ is its spin quantum number.

It is well known, that in these experiments only the traceless part of $V_{i j}$ may be determined. It is, therefore, as a matter of convenience customary to assume for $V_{i j}$ in (1) that

$$
V_{i i}=0 \text {. }
$$

We also note that

$$
V_{i j}=V_{j i} \text {. }
$$

We shall now calculate the diagonal sum of the Hamiltonian operator raised to some integral power. We shall write

$$
S_{n}=\operatorname{Tr}\left\{\mathfrak{H}^{n}\right\} .
$$

The general form of $S_{n}$ may be constructed by using the following two principles:

(a) Only terms with $\nu_{0}$ raised to an even power will appear (corresponding to the fact that experimentally one can not discriminate between a given direction of the magnetic field and the opposite direction).

(b) As $\mathfrak{J C}^{n}$ is a scalar operator, $S_{n}$ will be a " $c$-number" scalar, i.e., only scalar invariants formed by combination of the tensor $V_{i j}$ and the vector $n_{i}$ can appear in the expression for $S_{n}$.

Let us find the general form of $S_{3}$ : According to (a), (1), and (6), only terms of the form $\nu_{0}^{2} \alpha$ and $\alpha^{3}$ can appear. The coefficient to $\nu_{0}^{2} \alpha$ must, due to (b), (1), and (6), be a linear combination of those scalars, which can be constructed by using the vector $n_{i}$ twice and the tensor $V_{i j}$ once. There is only one such scalar in the present situation, namely,

$$
n_{i} V_{i j} n_{j} .
$$

[The other possibility $n_{i} n_{i} V_{j j}$ gives zero on account of (4).] Analogously the coefficient to the term $\alpha^{3}$ contains the scalars which can be constructed by using the tensor $V_{i j}$ three times, the only possibility being

$$
V_{i j} V_{j s} V_{s i} \text {. }
$$

[The determinant of a symmetric, traceless secondorder tensor is proportional to the expression just given and a scalar like $V_{i j} V_{j i} V_{s s}$ is zero on account of (4).]

Thus $S_{3}$ must be of the form

$$
S_{3}=6 g_{2}(I) \nu_{0}^{2} \alpha n_{i} V_{i j} n_{j}+\frac{4}{3} g_{3}(I) \alpha^{3} V_{i j} V_{j s} V_{s i}
$$

where $g_{2}$ and $g_{3}$ are functions, yet to be determined, of the spin quantum number $I$. The choice of specific values for the numerical factors in (7) is made for convenience.

In the same way (and utilizing $n_{i} n_{i}=1$ ), it is easily shown that $S_{2}$ must be of the form

$$
S_{2}=g_{1}(I) \nu_{0}^{2}+\frac{2}{3} g_{4}(I) \alpha^{2} V_{i j} V_{j i} .
$$

For $S_{1}$ we have

$$
S_{1}=0
$$

To determine the functions $g_{1} \cdots g_{4}$ we make a specific choice for $V_{i j}$ and $n_{i}$. Putting $n_{1}=n_{2}=0$, $n_{3}=1$ and $V_{11}=V_{22}=-1, V_{33}=2$ together with $V_{i j}=0$ for $i \neq j$ (thus $V_{i j} V_{j i}=6, V_{i j} V_{j s} V_{s i}=6$, and $n_{i} V_{i j} n_{j}=2$ ) gives, according to (1):

$$
\mathfrak{C}=-\nu_{0} I_{3}+\frac{1}{3} \alpha\left[3 I_{3}{ }^{2}-\left(I_{1}{ }^{2}+I_{2}{ }^{2}+I_{3}{ }^{2}\right)\right] .
$$

In a representation where the operators $I_{3}$ and $I_{1}{ }^{2}+$ $I_{2}{ }^{2}+I_{3}{ }^{2}$ are both diagonal, $S_{2}$ and $S_{3}$ are then easily calculated, and by comparison with (7) and (8), we obtain

$$
\begin{gathered}
g_{1}(I)=\frac{1}{3} I(I+1)(2 I+1), \\
g_{2}(I)=g_{4}(I)=\frac{1}{60}(2 I-1) g_{1}(I)(2 I+3), \\
g_{3}(I)=\frac{1}{42}(2 I-3) g_{2}(I)(2 I+5) ;
\end{gathered}
$$

(7) and (8), when written in the system of principal axes of $V_{i j}$ are of course identical to the results of Parker and Brown. ${ }^{2}$

Higher moments may be calculated along the same lines as indicated above with a few more complications, but as discussed below a knowledge of $S_{2}$ and $\mid S_{3}$ will normally be enough for the determination of $V_{i j}$.

\section{APPLICATIONS}

We shall consider the usual experimental setup, where the crystal can be rotated about, say, the $X$ axis, which is perpendicular to the direction of the magnetic field $H_{0}$. The angle between $H_{0}$ and the $Y$ axis is denoted by $\theta_{x}$ (measured positive counterclockwise looking down the $X$ axis), so that the unit vector $\bar{n}$ is given by $\left(0, \cos \theta_{x},-\sin \theta_{x}\right)$. Equations ( 7$)$ and (8) then take the form

$$
\begin{aligned}
& S_{2}=g_{1}(I) \nu_{0}^{2}+g_{2}(I) \nu_{Q}^{2}\left(1+\frac{1}{3} \eta^{2}\right) \\
& S_{3}=3 g_{2}(I) \nu_{0}^{2} \nu_{Q}\left[\phi_{y y}+\phi_{z z}+\left(\phi_{y y}-\phi_{z z}\right)\right. \\
& \left.\quad \times \cos 2 \theta_{x}-2 \phi_{y z} \sin 2 \theta_{x}\right]+g_{3}(I) \nu_{Q}{ }^{3}\left(1-\eta^{2}\right)
\end{aligned}
$$

Here

$$
\nu_{Q}=[3 / 2 I(2 I-1)]\left(e^{2} Q q / h\right),
$$

and

$$
\phi_{i j}=(1 / e q) V_{i j},
$$

where $e q$ and $\eta$ have their usual meaning. ${ }^{1}$

The dimensionless tensor $\phi_{i j}$ satisfies the following conditions:

$$
\begin{aligned}
& \phi_{i i}=0, \\
& \phi_{i j} \phi_{j i}=\frac{3}{2}\left(1+\frac{1}{3} \eta^{2}\right), \\
& \phi_{i j} \phi_{j s} \phi_{s i}=\frac{3}{4}\left(1-\eta^{2}\right) \text {, } \\
& \text { || } \phi_{i j} \|=\frac{1}{4}\left(1-\eta^{2}\right) .
\end{aligned}
$$


equations. This assumption means that (in the language of the usual methods) second-order shifts are observable. It should, however, be stressed that the present method requires that the full spectrum must be observable, in order to be able to construct the energy level scheme and from this calculate $S_{2}$ and $S_{3}$.

(a) If one principal axis of $\phi_{i j}$ is known in advance we choose this as the $X$ axis (giving $\phi_{x y}=\phi_{x z}=0$ ) and obtain from the rotation pattern about this axes [vide (8a) and (7a)] the values of:

$$
\begin{gathered}
\nu_{Q}\left(\phi_{y y}-\phi_{z z}\right), \\
\nu_{Q} \phi_{y z}, \\
3 g_{2}(I) \nu_{0}{ }^{2} \nu_{Q}\left(\phi_{y y}+\phi_{z z}\right)+4 g_{3}(I) \nu_{Q}^{3}\left\|\phi_{i j}\right\|,
\end{gathered}
$$

and

$$
\nu_{Q}^{2} \phi_{i j} \phi_{j i} .
$$

This gives four equations for the three unknown quantities $\nu_{Q} \phi_{y y}, \nu_{Q} \phi_{z z}$, and $\nu_{Q} \phi_{y z}$ (we have $\phi_{x x}=-\phi_{y y}-$ $\left.\phi_{z z}\right)$. It is readily seen that this determines these quantities uniquely (and that a certain overdetermination exists, giving a check on the experimental data). Thus knowing in full the tensor $\nu_{Q} \phi_{i j}$, the location of the principal axes in the $Y-Z$ plane as well as $\nu_{Q}$ and $\eta$ are then found by standard methods.

(b) If nothing is known in advance about $\phi_{i j}$, we shall indicate two methods to obtain first $\nu_{Q}$ and $\eta$.

(1) If three spectra, with the magnetic field in the $X, Y$ and $Z$ direction, respectively, are measured we see from (7a) and (11) that

$$
S_{3}\left(\theta_{x}=0\right)+S_{3}\left(\theta_{y}=0\right)+S_{3}\left(\theta_{z}=0\right)=3 g_{3}(I) \nu_{Q}{ }^{3}\left(1-\eta^{2}\right) .
$$

From this and the value of $\nu_{Q}{ }^{2}\left(1+\frac{1}{3} \eta^{2}\right)$ [from (8a)], $\nu_{Q}$ and $\eta$ may be obtained separately. Note that this result is correct for an arbitrary choice of three mutually perpendicular axes.

(2) If $S_{3}$ is observed for two different values of the magnetic field (the crystal being arbitrary but fixed in orientation) we get from (7a) (in an obvious notation)

$$
\left(\nu_{0}{ }^{\prime \prime} / \nu_{0}{ }^{\prime}\right)^{2}{S_{3}}^{\prime}-S_{3}{ }^{\prime \prime}=\left[\left(\nu_{0}{ }^{\prime \prime} / \nu_{0}{ }^{\prime}\right)^{2}-1\right] g_{3}(I) \nu_{Q}{ }^{3}\left(1-\eta^{2}\right),
$$

giving the value of $\nu_{Q}{ }^{3}\left(1-\eta^{2}\right)$, which combined with the value of $\nu_{Q}^{2}\left(1+\frac{1}{3} \eta^{2}\right)$ from (8a) gives $\nu_{Q}$ and $\eta$.

Knowing $\nu_{Q}$ and $\eta$, a rotation diagram about the $X$ axis will now, according to (7a) and (11) give $\phi_{y y}$, $\phi_{z z}, \phi_{x x}=-\phi_{y y}-\phi_{z z}$ and $\phi_{y z}$. Substituting these values in (12) and (13b) gives two equations connecting the still unknown components $\phi_{x y}$ and $\phi_{x z}$. In a $\phi_{x y}-\phi_{x z}$ plane these equations describe a circle and an ellipse, both centered in $(0,0)$. Of the four possible pair of values for $\left(\phi_{x y}, \phi_{x z}\right)$ a unique pair may be selected by the results of a single measurement with the magnetic field in the $X-Y$ or $X-Z$ plane. In practice a complete rotation pattern about the $Y$ axis (say) may be preferred, giving quite an overdetermination of the problem.

Our thanks are due to A. Pedersen and to A. Nielsen for commenting on the manuscript.

THE JOURNAL OF CHEMICAL PHYSICS

VOL UME 37 , N UM B E R 1

J U L Y 1, 1962

\title{
Proton Magnetic Resonance Spectrum of HDO ${ }^{*}$
}

\author{
John R. Holmes, $\ddagger$ Daniel Kivelson, $\$$ and William C. Drinkard" \\ Department of Chemistry, University of California, Los Angeles 24, California
}

(Received January 24, 1962)

\begin{abstract}
The proton magnetic resonance spectrum of the distinct species HDO has been observed in dilute solutions of $\mathrm{H}_{2} \mathrm{O}$ and $\mathrm{D}_{2} \mathrm{O}$ in carefully purified organic solvents. The $\mathrm{HDO}$ triplet is centered $0.030 \pm 0.003 \mathrm{ppm}$ upfield from the $\mathrm{H}_{2} \mathrm{O}$ resonance. The proton-deuteron spin-spin coupling constant $A_{\mathrm{HD}}$ is $1.1 \pm 0.1 \mathrm{cps}$. Approximate theories which provide explanations for the coupling constant and chemical shift are discussed.
\end{abstract}

\section{RESULTS}

$\mathbf{T}$ HE proton magnetic resonance spectrum of HDO has not been observed previously, primarily because the rapid exchange of protons and deuterons in

$\dagger$ Supported in part by the Research Corporation.

* Presented at the Southern California Regional meeting of the American Chemical Society, Los Angeles, December 1960.

I Present address: Research Laboratory, General Chemical Division, Allied Chemical Corporation, Morristown, New Jersey. $\S$ Alfred P. Sloan Fellow.

II Present address: Fabrics and Finishes Division, Experimental Station, E. I. du Pont de Nemours and Company, Wilmington, Delaware.
$\mathrm{H}_{2} \mathrm{O}-\mathrm{D}_{2} \mathrm{O}$ mixtures effectively averages the small differences in magnetic environment for protons in $\mathrm{H}_{2} \mathrm{O}$ and $\mathrm{HDO}$. However, the exchange rate can be slowed down considerably if the $\mathrm{H}_{2} \mathrm{O}-\mathrm{D}_{2} \mathrm{O}$ mixture is diluted with a solvent of low basicity which has been carefully purified. At sufficiently high dilution, then, one might expect to observe the spectrum of the mixed isotopic species HDO. Figure 1 (b) is the proton resonance spectrum in the water region of an acetone solution containing equimolar amounts of $\mathrm{H}_{2} \mathrm{O}$ and $\mathrm{D}_{2} \mathrm{O}$ at a total concentration of 1.1 mole/liter. This unsymmetrical multiplet is a superposition of the spectra of the 